\title{
Clinico-biochemical relation of Vitamin D3 with the severity of atopic dermatitis and response to supplementation of Vitamin D3: A randomized controlled trial
}

\author{
Narayan Prasad Modi ${ }^{1}$, Arun Kumar Dash ${ }^{2}$ \\ From ${ }^{1}$ Associate Professor, ${ }^{2} 3^{\text {rd }}$ Year Post Graduate, Department of Pediatrics, S.C.B. Medical College Hospital, Cuttack, Odisha, India
}

\begin{abstract}
Background: Atopic dermatitis (AD), also known as eczema, is one of the most common skin disorders among children and adults, with a steep rise in diagnoses among children. Many studies have investigated the relationship between Vitamin D3 (Vit D3) and AD. Methods: A randomized controlled trial was conducted among 60 children at SCB Medical College, Cuttack from August 2014 to November 2016. Children were randomly assigned to an intervention group that received 60,000 IU of Vit. D3 every week for 6 weeks in addition to regular treatment for $\mathrm{AD}$ and a control group that received same treatment of AD except for Vit. D3. Both the groups were followed up at 4 weeks and 8 weeks. Results: In 60 cases of moderate to severe AD, 70\% of the patients were male. About $81.7 \%$ of patients were from urban areas and $56.7 \%$ belonged to a middle socioeconomic class. In $76.6 \%$ of cases, family history of atopy was present. At baseline, mean SCORAD was $47.8 \pm 7.5$ in the intervention group, and $49.2 \pm 10.3$ in the control group. At baseline, serum Vitamin. D3 level (ng/ml) was 17.6 \pm 1.8 in intervention and 17.3 \pm 3.5 in control group. After Vitamin. D3 supplementation, the SCORAD improved to $12.8 \pm 5.1$ (75\% reduction) at 4 weeks, and $3.6 \pm 2.1$ ( $92 \%$ reduction) at 8 weeks in the intervention group. In the control group, the mean SCORAD was $18.8 \pm 9.1$ (61\% reduction) at 4 weeks, and $7.3 \pm 4$ ( $85 \%$ reduction) at 8 weeks. Discussion: The improvement of serum 25-hydroxy Vit. D3 was $134 \%$ and $366 \%$ in the intervention group compared to $78 \%$ and $121 \%$ in the observation group after 4 weeks and 8 weeks respectively. The p-values at both the time points were significant $(<0.05)$ for the intervention group as compared to the control group. Conclusion: Short-term therapeutic supplementation of Vitamin. D3 in children with moderate to severe AD improves the clinical score. There is an inverse relationship between serum Vitamin. D3 level and severity of AD.
\end{abstract}

Key words: Atopy, Eczema, Vitamin D3, SCORAD, Pediatric

A topic dermatitis (AD), also known as Eczema, is one of the most common skin disorders among children and adults, with a steep rise in prevalence among children [1,2]. Up to $20 \%$ of children are now affected by AD in developing countries. AD is a chronic and relapsing disease that is associated with inflammatory processes often preceding asthma and allergic rhinitis with a prevalence of 9-20\% worldwide [3]. AD is characterized by dry and extremely pruritic skin [4]. This leads to the appearance of erythematous scaly patches, excoriation, and lichenification of skin. Severity of $\mathrm{AD}$ can range from a few xerotic patches on the skin to involvement of the entire body causing intense psychological distress [5]. Due to defects in the innate immune system, patients with $\mathrm{AD}$ have a greater chance of acquiring infection by microbial organisms such as Staphylococcus aureus and herpes simplex virus.

\section{Access this article online}

Received - 07 December 2021

Initial Review - 20 December 2021

Accepted - 12 December 2021

DOI: $10.32677 /$ ijch.v8i12.3227
Treatment strategies have often focused on allergen avoidance and topical application of medications. But recently, research has focused on its connection with immune function and the skin's natural barrier function. AD is not only associated with defects in the immune system but also these defects worsening the inflammation [6]. Within the past 5 years, focus has moved from the known interactions of Vitamin D3 (Vit. D3), bone mineralization, and osteoporosis to a broader role including cardiovascular, endocrine, cancers, and chronic diseases such as hypertension [7].

Vit. D3 is a fat-soluble vitamin. Under ultraviolet rays' exposure, it is synthesized under the skin. It is also found in natural foods as well as supplemental diets. Vitamin. D3 also has an important role in immunity, as it improves innate and adaptive immune responses by altering local calcium balance and by binding to nuclear vitamin receptors, which regulate gene transcription [8]. Vitamin. D3 regulates the activity of various immune cells such as monocytes, dendritic cells, and lymphocytes as well as regulates the function of epithelial cells which are

Correspondence to: Dr. Narayan Prasad Modi, Department of Pediatrics, S.C.B. Medical College Hospital, Cuttack, Odisha, India. E-mail: npm234@gmail.com

(C) 2021 Creative Commons Attribution-NonCommercial 4.0 International License (CC BY-NC-ND 4.0). 
important in allergic inflammation [9]. The levels of Vit. D3 often varies among people of different skin shades, geographic locations, sun exposure, diet, and hereditary factors.

Many studies have investigated the relationship between Vitamin. D3 and AD. The severity of AD tends to increase in patients who have lower levels of Vitamin. D3 [10]. Pilot studies have revealed a possible correlation between the severity of AD and very low serum Vitamin. D3 levels. and. The severity of the disease improved after Vitamin. D3 supplementation, according to SCORAD.

\section{MATERIALS AND METHODS}

The study was conducted at SCB Medical College, Cuttack from August 2014 to November 2016. Ethical approval for the study was granted by the hospital ethics committee. An informed written consent was obtained from parents of all subjects prior to enrolment in the study.

Subjects between the age group of 1-14 years diagnosed with $\mathrm{AD}$ by a dermatologist as per Hanifin and Rajka criteria and subjects willing to give blood for estimation of Vitamin. D3 levels were included in the study. The SCORAD was divided into three grades of severity: mild ( $<25$ points), moderate $(25-50$ points), and severe ( $>50$ points) [11]. Only moderate to severe cases of $A D$ as per SCORAD were eligible for inclusion in this study. Serum Vitamin. D3 levels were measured in children with moderate to severe $\mathrm{AD}$, and categorized as: sufficient $(>30$ $40 \mathrm{ng} / \mathrm{ml}$ ), insufficient (20-30 ng/ml), and deficient (20 ng/ml). Subjects with other atopic conditions (asthma, allergic rhinitis, conjunctivitis) or other skin diseases, syndromes, underlying systemic co-morbidity, and severe malnutrition were excluded from the study.

The intervention group received 60,000 IU of Vitamin. D3 supplementation every week for 6 weeks in addition to the standard treatment of $\mathrm{AD}$ as advised by a dermatologist. Control group received the same treatment of AD except for Vitamin. D3. Both groups were followed up at 4 weeks and 8 weeks to measure the response. Baseline data and socio-demographic parameters were recorded in a pre-designed data collection form. The following outcomes were measured in both the groups;

1. Serum 25-dihydroxy cholecalciferol level (Vit. D3)

2. Response to Vitamin. D3 supplementation in children with moderate to severe AD.

Children were randomly divided into two groups (intervention and control group) by a computer-generated random number table. Allocation concealment was done by the use of sealed, opaque envelopes. Patients and investigators were blinded to the identity of the study drug.

Data were entered into Microsoft excel sheet 2016 version. Statistical analysis was carried out using STATA software version 16.0 (USA). Data were analyzed using Shapiro-Wilk test for normality distribution. Continuous data were analyzed using "t-test," and categorical data using "Chi-square test." A p $<0.05$ was considered to be statistically significant.

\section{RESULTS}

A total of 86 children were assessed for eligibility. After excluding 22 subjects not meeting the inclusion criteria, 60 children with moderate to severe $\mathrm{AD}$ were finally randomized into two groups: intervention and control group (30 in each group) (Fig. 1). The baseline parameters of the two groups were comparable (Table 1). Out of all the subjects, $70 \%$ were male. About $81.7 \%$ of patients were from urban areas and $56.7 \%$ belonged to a middle socioeconomic class. In $76.6 \%$ of subjects, family history of atopy was present. At baseline, mean SCORAD was 47.8 \pm 7.5 in the intervention group, and $49.2 \pm 10.3$ in the control group. At baseline, serum Vitamin. D3 level ( $\mathrm{ng} / \mathrm{ml})$ was $17.6 \pm 1.8$ in the intervention and $17.3 \pm 3.5$ in the control group.

After Vitamin. D3 supplementation, the SCORAD improved to $12.8 \pm 5.1$ ( $75 \%$ reduction) at 4 weeks, and $3.6 \pm 2.1(92 \%$ reduction) at 8 weeks in the intervention group. In the control group, the mean SCORAD was $18.8 \pm 9.1$ (61\% reduction) at 4 weeks, and $7.3 \pm 4$ ( $85 \%$ reduction) at 8 weeks. The improvement of serum 25-hydroxy Vit. D3 was 134,366 in the intervention group compared to 78,121 in the observation group after 4 weeks and 8 weeks respectively. The p-value at both the time points was significant $(<0.05)$ for the intervention group compared to the control group (Table 2 and 3). There was no report of any adverse event in either of the groups.

\section{DISCUSSION}

$\mathrm{AD}$ is a chronic, pruritic disease of the skin which is relapsing in nature. There is a steep rise in incidence of $\mathrm{AD}$ during the past 2 decades, more so in children. Various studies have been conducted to examine the incidence and epidemiological profile of $\mathrm{AD}$ in different geographies with respect to gender, rural and urban distribution, socio-economic status, and family history of atopy. Recent studies have highlighted the relationship of AD with that of Vitamin. D3. An inverse relationship between the severity of AD and serum Vitamin. D3 has been shown by many studies.

In the present study, we found a significant improvement in the SCORAD after supplementation with Vitamin. D3 in children with moderate to severe AD. There was no report of any adverse events. The male-to-female ratio was 3:1 in our study. In a study

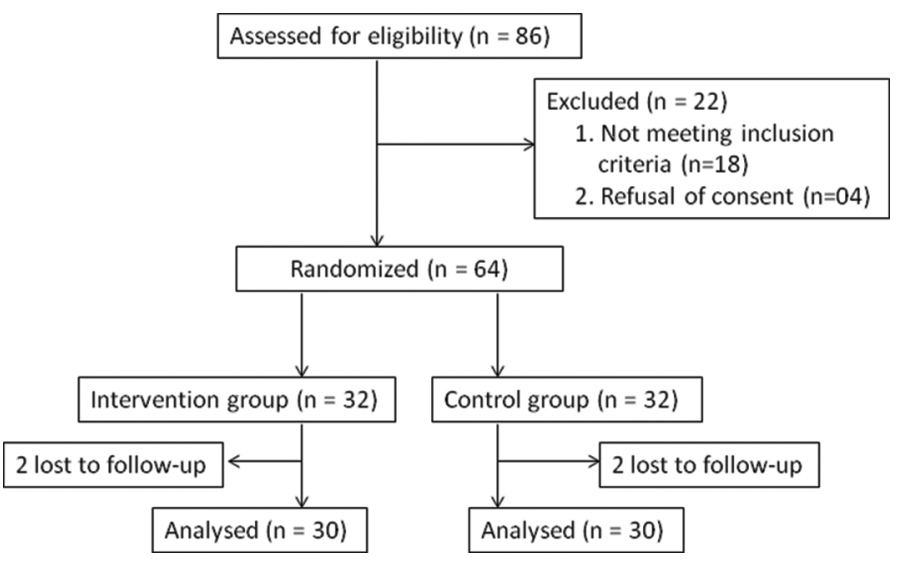

Figure 1: Study flow chart showing patient flow in the study 
Table 1: Baseline characteristics of study children with moderate to severe AD as per SCORAD

\begin{tabular}{|c|c|c|c|}
\hline Variables & Intervention group $(\mathrm{n}=\mathbf{3 0})$ & Control group $(n=30)$ & p-value \\
\hline Age, year (mean \pm SD) & $7.4 \pm 5.8$ & $7.7 \pm 5.6$ & 0.24 \\
\hline Male sex, n (\%) & $17(56.7)$ & $16(53.3)$ & 0.53 \\
\hline Urban area, n (\%) & $14(46.7)$ & $15(50)$ & 0.78 \\
\hline H/o atopy in family, $\mathrm{n}(\%)$ & $11(36.7)$ & $10(33.3)$ & 0.47 \\
\hline Lower socio-economic status, n (\%) & $9(30)$ & $7(23.3)$ & 0.29 \\
\hline Moderate malnutrition, $\mathrm{n}(\%)$ & $6(20)$ & $5(16.7)$ & 0.18 \\
\hline H/o exclusive breast feeding, $\mathrm{n}(\%)$ & $19(63.3)$ & $21(70)$ & 0.17 \\
\hline
\end{tabular}

AD: Atopic dermatitis

Table 2: Vitamin D3 level and SCORAD in both groups at various time points

\begin{tabular}{lccccc}
\hline Time points & \multicolumn{2}{c}{ Intervention group $(\mathbf{n}=\mathbf{3 0})$} & & \multicolumn{2}{c}{ Control group $(\mathbf{n}=\mathbf{3 0})$} \\
\cline { 2 - 3 } & $\begin{array}{c}\text { SCORAD, } \\
\text { mean } \pm \text { SD }\end{array}$ & $\begin{array}{c}\text { Vitamin D3 level } \\
(\mathbf{n g} / \mathbf{m l}), \text { mean } \pm \text { SD }\end{array}$ & & $\begin{array}{c}\text { SCORAD, } \\
\text { mean } \pm \text { SD }\end{array}$ & $\begin{array}{c}\text { Vitamin D3 level } \\
(\mathbf{n g} / \mathbf{m l}), \mathbf{m e a n} \pm \text { SD }\end{array}$ \\
\hline At admission & $47.8 \pm 7.5$ & $17.6 \pm 1.8$ & & $49.2 \pm 10.3$ & $17.3 \pm 3.5$ \\
At 4 weeks & $12.8 \pm 5.1$ & $41.3 \pm 6.4$ & & $18.8 \pm 9.1$ & 0.26 \\
At 8 weeks & $3.6 \pm 2.1$ & $83.1 \pm 8.8$ & & $7.3 \pm 4.0$ & $0.03^{*}$ \\
\hline
\end{tabular}

*p<0.05, SD: Standard deviation

Table 3: Percentage (\%) reduction of SCORAD in intervention and control group at various time points

\begin{tabular}{lccc}
\hline Time points & $\begin{array}{c}\text { Intervention } \\
\text { group }(\mathbf{n}=\mathbf{3 0})\end{array}$ & $\begin{array}{c}\text { Control } \\
\text { group }(\mathbf{n}=\mathbf{3 0})\end{array}$ & p-value \\
\hline At admission & 100 & 100 & 0.26 \\
At 4 weeks & 75 & 61 & $0.04 *$ \\
At 8 weeks & 92 & 85 & $0.012^{*}$ \\
\hline${ }^{*} \mathbf{p}<\mathbf{0 . 0 5}$
\end{tabular}

*p $<0.05$

from North India, the male-to-female ratio was 1.6:1 [12]. Our study also revealed an increased prevalence of AD in males in accordance with a study from China which also indicated male dominance [13]. In our study, urban children constituted $81.7 \%$ and rural children constituted $18.3 \%$ with the ratio being $4.45: 1$. In another study, the prevalence of $\mathrm{AD}$ as per ratio of urban to rural was found to be 5:1 [14]. The cause of this high variation was depicted as rapid industrialization, change in lifestyle, and dietary habits.

We also found out in our study that $33 \%$ of children had a positive family history of atopy (eczema, asthma, or allergic rhinitis). According to a previous study, AD is 2.9 times more likely to occur in children of atopic parents than those of nonatopic parents [15]. This study also depicted maternal atopy having the strongest (20-26\%) influence on childhood AD. When both the parents are affected, the odds ratio increases to 1.75. Nearly $27 \%$ of children belonged to lower socioeconomic status. This is in line with a previous systematic review that showed AD is more common among people with higher economic status [16]. The possible reason being hygiene hypothesis which states that higher exposure to infection in early life is a protective factor for AD. Therefore, the prevalence of AD with relation to socioeconomic status remains controversial.

In our study, the intervention group showed both improvements in SCORAD and Vit. D3 compared to observation group with $\mathrm{p}<0.05$. An earlier randomized clinical trial on the effectiveness of Vitamin. D3 supplementation in the treatment of AD found significant improvements in SCORAD across all the severity of AD [1]. The authors used Vitamin. D3 supplementation for 60 days compared to 6 weeks in our study. However, our findings were in accordance with this study.

Our study has an important implication, as a long-term supplementation might increase the risk of Vit. D3 toxicity without any additional benefits. Albenali et al. reported an improvement in SCORAD after taking Vit. D3 for 2 months by increasing IL-37 level [17]. SCORAD was also found to be improved upon supplementation with Vit. D3 in another study [18]. Contrastingly, one study found a link between elevated intake of Vit. D3 during infancy and increased incidence of $\mathrm{AD}$ in children younger than 6 years [11].

Though our study has several strengths, it has few limitations also. The limitations were: (a) small sample size, (b) the dose and duration of Vit. D3 was arbitrary; (c) neither allergic nor immunological parameters were studied to determine the mechanism of Vit. D3 action in AD.

\section{CONCLUSION}

Short-term therapeutic supplementation of Vit. D3 in children with moderate to severe AD improves the clinical score. There is an inverse relationship between serum Vit. D3 level and severity of AD. However, it remains to be seen if further studies examining different doses and duration regimens will confirm or refute the current study findings.

\section{REFERENCES}

1. Amestejani M, Salehi BS, Vasigh M, Sobhkhiz A, Karami M, Alinia H, et al. Vitamin D supplementation in the treatment of atopic dermatitis: A clinical trial study. J Drugs Dermatol 2012;11:327-30.

2. Sidbury R, Sullivan AF, Thadhani RI, Camargo CA Jr. Randomized controlled trial of Vitamin D supplementation for winter-related atopic 
dermatitis in Boston: A pilot study. Br J Dermatol 2008;159:245-7.

3. Flohr C, Mann J. New approaches to the prevention of childhood atopic dermatitis. Allergy 2014;69:56-61.

4. Traidl S, Werfel T, Traidl-Hoffmann C. Atopic eczema: Pathophysiological findings as the beginning of a new era of therapeutic options. Handb Exp Pharmacol 2022;268:101-15.

5. Vakharia PP, Silverberg JI. Adult-onset atopic dermatitis: Characteristics and management. Am J Clin Dermatol 2019;20:771-9.

6. Spergel JM. Epidemiology of atopic dermatitis and atopic march in children. Immunol Allergy Clin North Am 2010;30:269-80.

7. Vestita M, Filoni A, Congedo M, Foti C, Bonamonte D. Vitamin D and atopic dermatitis in childhood. J Immunol Res 2015;2015:257879.

8. Mansour NO, Mohamed AA, Hussein M, Eldemiry E, Daifalla A, Hassanin S, et al. The impact of Vitamin D supplementation as an adjuvant therapy on clinical outcomes in patients with severe atopic dermatitis: A randomized controlled trial. Pharmacol Res Perspect 2020;8:e0679.

9. Bartley J. Vitamin D: Emerging roles in infection and immunity. Expert Rev Anti Infect Ther 2010;8:1359-69.

10. Lombardi C, Passalacqua G; Italian Vitamin D Allergy Group. Vitamin D levels and allergic diseases. An Italian cross-sectional multicenter survey. Eur Ann Allergy Clin Immunol 2017;49:75-9.

11. Bäck O, Blomquist HK, Hernell O, Stenberg B. Does Vitamin D intake during infancy promote the development of atopic allergy? Acta DermVenereol 2009;89:28-32.

12. Dhar S, Kanwar AJ. Epidemiology and clinical pattern of atopic dermatitis in a North Indian pediatric population. Pediatr Dermatol 1998;15:347-51.

13. Wang X, Li LF, Zhao DY, Shen YW. Prevalence and clinical features of atopic dermatitis in China. Biomed Res Int 2016;2016:2568301.
14. Sánchez-Armendáriz K, García-Gil A, Romero CA, Contreras-Ruiz J, Karam-Orante M, Balcazar-Antonio D, et al. Oral vitamin D3 5000 IU/day as an adjuvant in the treatment of atopic dermatitis: A randomized control trial. Int J Dermatol 2018;57:1516-20.

15. Hata TR, Audish D, Kotol P, Coda A, Kabigting F, Miller J, et al. A randomized controlled double-blind investigation of the effects of Vitamin D dietary supplementation in subjects with atopic dermatitis. J Eur Acad Dermatol Venereol 2014;28:781-9.

16. Uphoff E, Cabieses B, Pinart M, Valdés M, Antó JM, Wright J. A systematic review of socioeconomic position in relation to asthma and allergic diseases. Eur Respir J 2015;46:364-74.

17. Albenali LH, Danby S, Moustafa M, Brown K, Chittock J, Shackley F, et al. Vitamin D and antimicrobial peptide levels in patients with atopic dermatitis and atopic dermatitis complicated by eczema herpeticum: A pilot study. J Allergy Clin Immunol 2016;138:1715-9.

18. Javanbakht MH, Keshavarz SA, Djalali M, Siassi F, Eshraghian MR, Firooz A, et al. Randomized controlled trial using Vitamins E and D supplementation in atopic dermatitis. J Dermatol Treat 2011;22:144-50.

Funding: None; Conflicts of Interest: None Stated.

How to cite this article: Modi NP, Dash AK. Clinico-biochemical relation of Vitamin D3 with severity of atopic dermatitis and response to supplementation of Vitamin D3: A randomized controlled trial. Indian J Child Health. 2021; 8(12):412-415. 